\title{
Deep Micro Vibrotactile, DMV and its Applications
}

\author{
Yasuhiro Suzuki \\ Graduate School of Informatics, Nagoya University, Furocho Chikusa \\ Nagoya City, Aichi Prefecture 464/0814, Japan* \\ E-mail: ysuzuki@nagoya-u.jp.com \\ www.ysuzuki.info
}

\begin{abstract}
Deep Micro Vibrotactile, DMV is infrasound. DMVs produce sound effects when mixed with audio. Since the DMV has a transcendental low frequency, mixing it with sound such as music does not affect the frequency spectrum in the audible range. However, through demonstration experiments at concerts, it was confirmed that DMV changes the tactile qualities of the sound. This paper introduces the acoustic features and case studies of DMVs
\end{abstract}

Keywords: Deep Micro Vibrotactile, Infrasonic, very low frequency sound, sound pressure analysis

\section{Introduction}

Infrasound is a sound generated by vibrations of 20 times or less per second. In general, the sound loses energy due to vibration as it passes through the medium. Therefore, high frequencies quickly lose energy and are attenuated, while low frequencies are less attenuated. Therefore, it can travel up to thousands of kilometres. Low frequencies are less directional than high frequencies. Proceed in concentric circles from the source. Even if there are obstacles, go around ${ }^{1)}$.

In the natural world, low-frequency sounds are generated from wind, air vibrations, self, volcanic activity, avalanches, waterfalls, signals from animals such as elephants, etc. Artificial objects arise from outdoor units of air conditioners, wind turbines for wind power generation, turbines of thermal power plants, machine tools, blasts, pipe organs, etc ${ }^{1)}$.

Infrasound is regarded as noise, and its effects on the human body and shielding methods have been investigated 1). Kuhn, et al. confirmed that infrasound near the hearing threshold might induce changes in neural activity.

Some of these brain regions are known to be involved in auditory processing, while others are regarded as key players in emotional and autonomic control ${ }^{2)}$. 
Low-frequency sounds have been used primarily in medicine. One of the most widely used techniques is Whole-body vibration (WBV). WBV is a therapeutic method that exposes the entire body to mechanical oscillations while the patient stands or sits on a vibrating platform. WBV appears to affect balance, muscle function positively, and physical performance, which would benefit the skeletal muscle's treatment, decline seen in sarcopenia ${ }^{3)}$.

In music compose, the lowest note on a pipe organ is 16Hz. The d-Moll Toccata and Fugue BWV 565, composed by Johann Sebastian Bach (1685-1750), contains a $16 \mathrm{~Hz}$ bass.

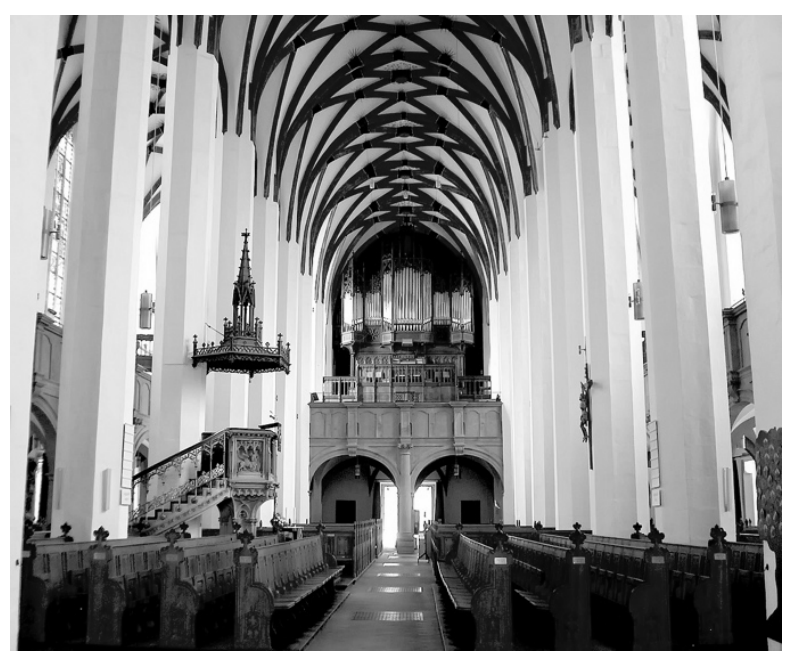

Fig. 1. St. Thomas Church, Leipzig and the Pipe-organ. J.S. Bach worked as a music director from 1723 until his death in 1750. From Wikipedia and this photograph (by S-Kay) is public domain

Inaudible low frequencies below $20 \mathrm{~Hz}$ distort the sound in the audible range. Therefore, infrasound is carefully removed in the production of music software. On the other hand, as mentioned earlier, in the Middle Ages, infrasonic was used in music as Bach used. Acoustic studies have shown that pipe organs can generate from $10 \mathrm{~Hz}$ to $10,000 \mathrm{~Hz}$ and above ${ }^{4)}$.

Pipe organs require 7-meter-long pipes to generate infrasound. Because the low-frequency wavelength is long. Also, because the weight is $150 \mathrm{Kg}$, the building sinks after the pipe organ is delivered. It will take a long time for the pipe organ to stop subsidence and the building from becoming structurally stable. Why did it generate inaudible low-frequency sound at such a cost?

Infrasound is inaudible. Besides, there are no known musical instruments that generate infrasound other than pipe organs. If there is an instrument that produces infrasound, it cannot produce audible sounds. Therefore, it is natural that music that actively uses infrasound has not been developed.

In contemporary music, some works use infrasound. They use electronically generated infrasound. Franco Schoeman focused on the low frequencies that elephants use for communication, and composed music using low frequencies. He has independently developed a notation for low-frequency composition ${ }^{5)}$.

In sound sources such as records, infrasound of $20 \mathrm{~Hz}$ or less has been removed. This is because if the sound source contains infrasound, the housing for music playback resonates, causing sound deterioration. The tonearm is a part that picks up sound on a turntable that plays records. The Tonearm has a record needle and makes physical contact with the record, so the record physically vibrates the Tonearm. The resonance between the vinyl record and the tonearm distorts the sound. It causes Tonearm resonance when included in a sound source of $20 \mathrm{~Hz}$ or less on a vinyl record ${ }^{6}$. Therefore, frequencies below $20 \mathrm{~Hz}$ have been removed.

\section{Add a super constant frequency to the sound source}

Fourier has shown that a wave with periodicity is the sum of sine waves of any complexity. The Fourier series of the periodic function $\mathrm{x}(\mathrm{t})$ with the interval $(-\mathrm{T} / 2, \mathrm{~T} / 2$ as one cycle can be described as follows because the

$$
\begin{aligned}
x(t)=\frac{a_{0}}{2}+ & a_{1} \cos (t)+b_{1} \sin (t)+a_{2} \cos (2 t) \\
& +b_{2} \cos (2 t)+\cdots=f(x) \\
& =a_{0} \\
& +\sum_{n=1}^{\infty}\left(a_{n} \cos \frac{2 n \pi t}{T}+b_{n} \sin \frac{2 n \pi t}{T}\right)
\end{aligned}
$$

waves to be added have orthogonality (Fig. 3). We observe the spectrograph of audible part (higher than $20 \mathrm{~Hz}$ ) does not change compared with the case when 
$15 \mathrm{~Hz}$ has added (Fig. 3). This is obvious from the Fourier analysis and pattern of spectrographs do not change.
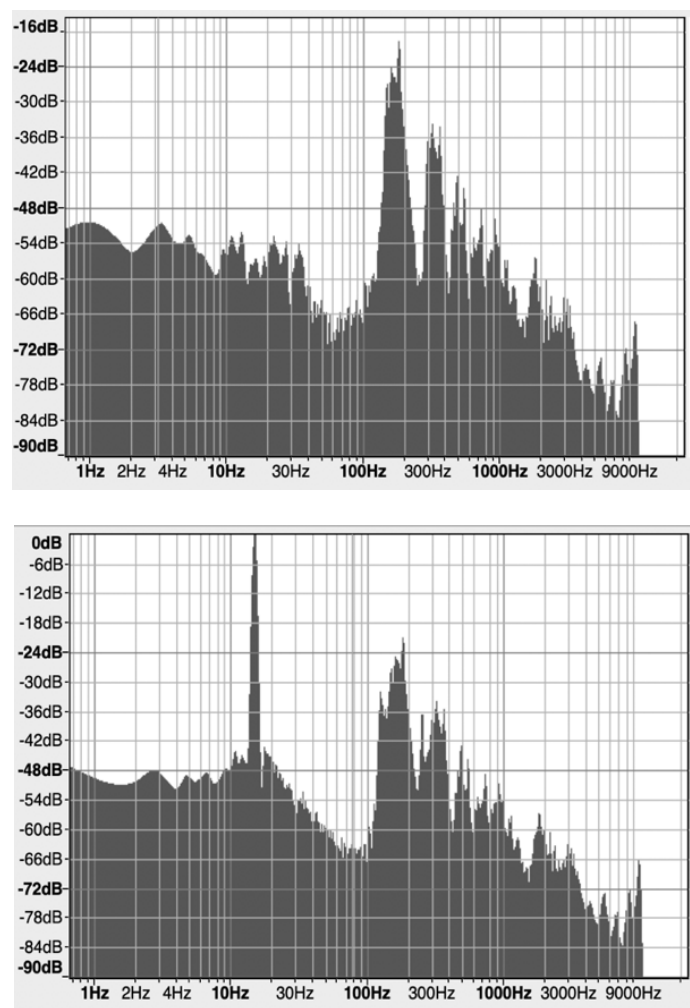

Fig. 2. Example of frequency spectrograph when infrasonic sound added to a sound. Upper: Frequency Spectrograph of No infrasound added, Lower: $15 \mathrm{~Hz}$ added to the sound. A steep peak of $15 \mathrm{~Hz}$ shows the added $15 \mathrm{~Hz}$. And the frequency spectrographs higher than $20 \mathrm{~Hz}$ show no differentiations. We used the female sample voice in Mexican, which is given as a sample of the Audition, which is software produced by Adobe Co. Ltd.

As mentioned above, low-frequency sound may induce physical vibration of the playback equipment. Therefore, when a sound source such as a record or Compact Disc, $\mathrm{CD}$ is expanded by Fourier series, waves of $20 \mathrm{~Hz}$ or less are not included. Therefore, adding infrasound of $20 \mathrm{~Hz}$ or less to a commercial sound source does not affect each wave of $20 \mathrm{~Hz}$ or more.

Add sounds below $20 \mathrm{~Hz}$ to sounds that do not include sounds below $20 \mathrm{~Hz}$ and analyze the frequency spectrum. The part of the frequency spectrum above $20 \mathrm{~Hz}$ does not change before and after adding sound below $20 \mathrm{~Hz}$.

\section{Method and Material}

We analyzed Schumann's second-movement -Symphony No 3 in E-flat major, Op 97, We analyzed the sound intensity by adding a $15 \mathrm{~Hz}$ sine wave to this sound source ${ }^{8)}$. Besides, frequency spectrum analysis was performed using Audacity 2.4.2.

\subsection{Acoustic sound pressure analysis}

We have proposed analyzing the time change of sound
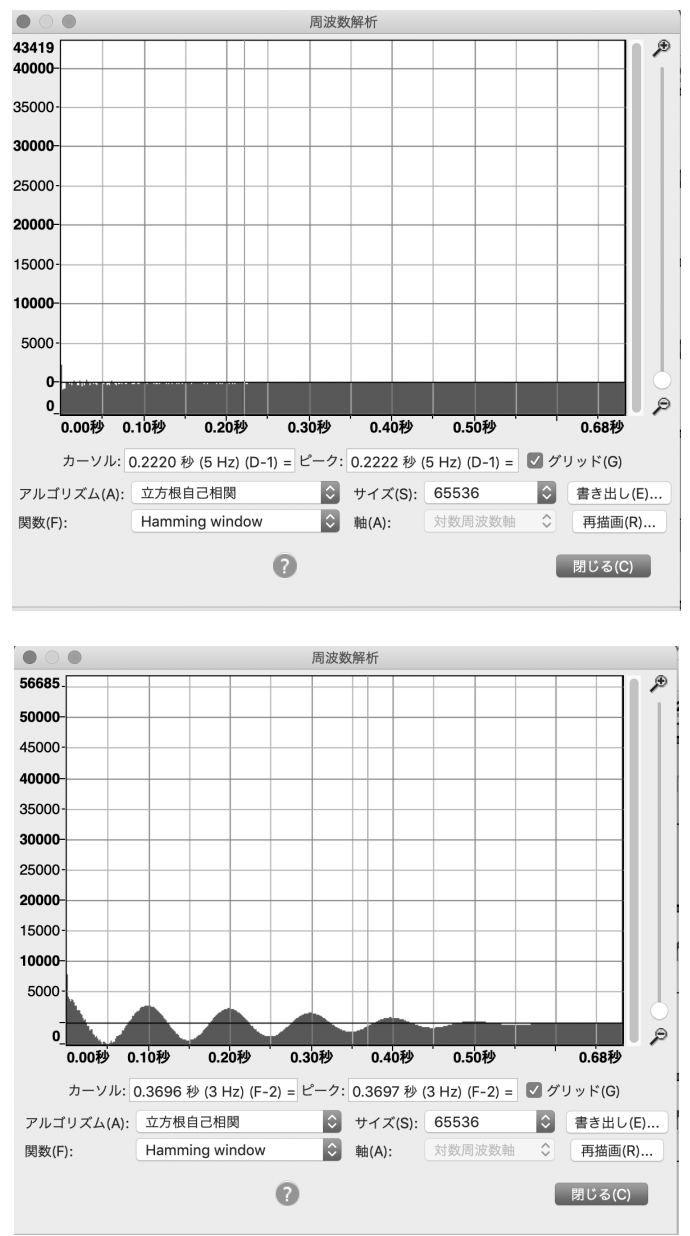

Fig. 3. Upper) Autocorrelation of Schumann's symphony, Bottom) Autocorrelation when $15 \mathrm{~Hz}$ is put in the same sound source. Autocorrelation is cube root autocorrelation.

pressure of voice data [8]. The method is described below. First, the sound pressure is discretized as follows.

$$
F(p)=p_{i}, \text { if }\left(b_{i} \leq p<b_{i+1}\right) \text {, }
$$



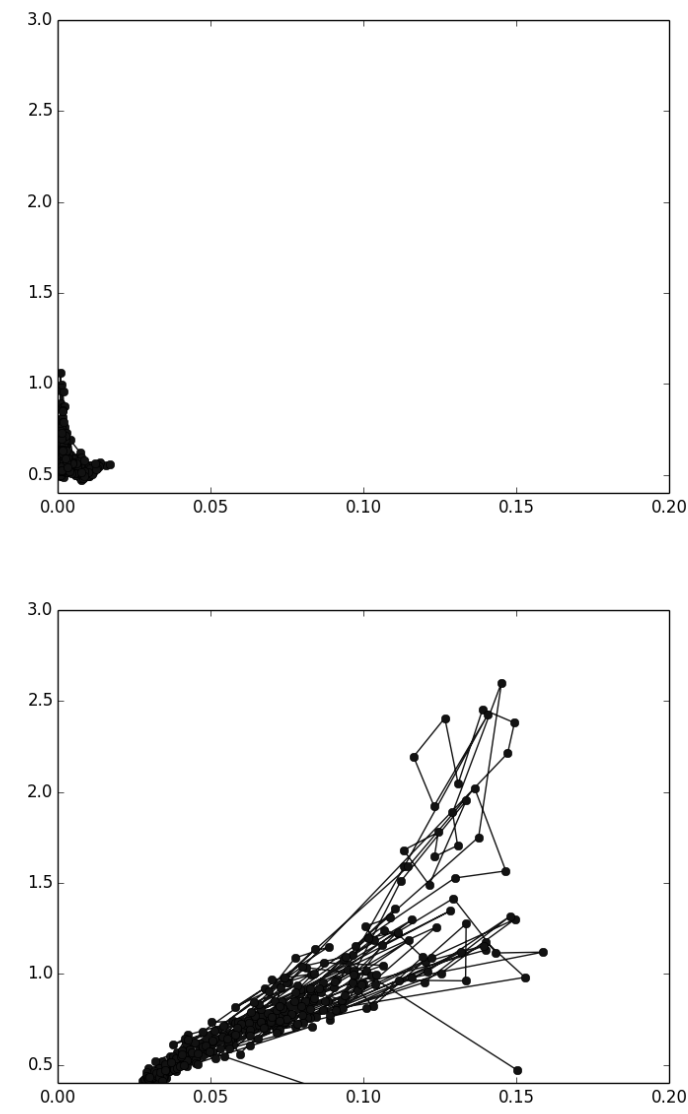

Fig. 4. Upper) Acoustic sound pressure analysis of Schumann's symphony, Bottom) Autocorrelation when $15 \mathrm{~Hz}$ is put in the same sound source.

where

$$
\beta=\frac{p_{M A X}-p_{\min }}{n}, b_{i+1}=b_{i}+\beta \text {. }
$$

Next, the sound source is divided into t seconds and averaged. Then, assign bi to pi of each t. From the above, the sound source is converted into a sequence of pi. Calculate the mean and standard deviation of this sequence of points. Then, plot the mean on the vertical axis and the standard deviation on the horizontal axis. Then, the time change of the intensity of the sound source is converted into a two-dimensional pattern.

Even if infrasonic was mixed with ordinary music, there was no difference in the audible part's frequency spectrum. However, when the autocorrelation was measured, it was confirmed that the autocorrelation had a structure when $15 \mathrm{~Hz}$ was mixed. Also, acoustic sound pressure analysis showed that mixing $15 \mathrm{~Hz}$ made a big difference.

So far, there has been little scientific evidence about the use of infrasonic. However, there are relatively many proven effects. For example, Dave of hard rock band Metallica5), Dave Pickerel focused on the $16 \mathrm{~Hz}$ used in Bach's "Toccata and Fugue" mentioned earlier. And he created a subwoofer that was about the size of a guitar case. Then, I selected a song that contained a lot of deep bass from their songs and outputs it from this subwoofer. The super-heavy bass was exposed to whiskey being brewed in a barrel. A whiskey appraiser has tasted the whiskey and confirmed that it is a good quality whiskey. Such examples are used in processes involving fermentation such as winemaking. However, little scientific research has been done.

This study showed that adding super-heavy bass to music causes a large change in the temporal change of acoustic sound pressure. This result opens up possibilities for the use of infrasonic, which was previously unknown.

\section{References}

1. Alfred J.Lotoka, Elements of Physical Biology, Williams and Wilkins Company, 1925.

2. Dimas Martins Afonso, Gjini Erida, Modeling Competitive Mixtures With the Lotka-Volterra Framework for More Complex Fitness Assessment Between Strains, Frontiers in Microbiology, 11, 2132, 2020.

3. Takabayashi J., Dicke M. (1997) Herbivore-Induced Plant Volatiles with Multifunctional Effects in Ecosystems: A Complex Pattern of Biotic Interactions. In: Abe T., Levin S.A., Higashi M. (eds) Biodiversity. Springer, New York, NY. https://doi.org/10.1007/978-1-4612-1906-4_9

4. Kerner, Edward H, Comment on Hamiltonian structures for the n-dimensional Lotka-Volterra equations, Journal of Mathematical Physics 38, 1218 (1997); https://doi.org/10.1063/1.5318751, Published Online: 04 June 1998

5. Metallica Talk Top-Secret Distilling Process Behind New 'Blackened' Whiskey, Rolling Stone, SEPTEMBER 15 $\underline{2018}$ 